\title{
Polyaniline in Situ Grafted to Graphene Sheets
}

\author{
Elliard Roswell S. Yanza', Geoffrey Matthew C. Tan ${ }^{1}$, Christine Joy U. Querebillo', \\ Armando S. Somintac ${ }^{2}$, Arnel A. Salvador ${ }^{2}$, and Erwin P. Enriquez ${ }^{*}$ \\ ${ }^{1}$ Department of Chemistry, School of Science and Engineering, Ateneo de Manila University, Loyola Heights, \\ Quezon City, Philippines \\ ${ }^{2}$ National Institute of Physics, University of the Philippines-Diliman, Diliman, Quezon City, Philippines
}

\begin{abstract}
Graphene is one of the most promising allotropes of carbon with wide applications in nanotechnology. Modification of graphene by chemical means can further expand its uses. Here, liquid-exfoliated graphene is functionalized with $p$-aminophenyl moiety using $p$ nitrophenyl diazonium salts which were diazotized in situ then reduced by tin (II) chloride. The aminophenyl-graphene thus produced is further modified to form polyanilinegraphene (PANI-GNH) by in situ oxidative graft polymerization of aniline using ammonium peroxydisulfate as oxidizing agent. The properties of the PANI-GNH were compared with polyaniline/graphene blends by Raman, infrared and UV-Visible spectroscopy, and cyclic voltammetry. Indeed, PANI-GNH registered different spectrochemical and electrochemical properties compared with the physically blended PANI and GNH, a manifestation of the effect of chemical grafting on the overall property of the modified graphene.
\end{abstract}

Keywords: graphene; polyaniline; diazonium salts; composite

\section{INTRODUCTION}

Graphene is a two-dimensional form of $s p^{2}$ hybridized carbon with atoms arranged in a honeycomb lattice. It is the basic building block for graphitic materials of all other dimensionalities (Wei et al., 2009; Wehling et al., 2008). It has high surface area, tensile strength, and thermal and electrical conductivity. It is flexible, transparent and has low coefficient of thermal expansion. It is a zero-band gap semiconductor and is chemically inert. It is considered as one of the most promising materials for post-silicon electronics because it combines high electron mobility with atomic thickness (Wei et al.,
2009; Wehling et al., 2008; Lu et al., 2009; Liu et al., Graphene, 2008; Li et al., Synthesis, 2012; Kumar et al., Polyaniline, 2012; Zhao et al., Effect, 2012; Li and Shi).

Polyaniline is an intrinsically conducting polymer (ICP) known for its facile synthesis, stability and reversible acid/base-doping/ dedoping (Li et al., Synthesis, 2012; Wu et al., Supercapacitors, 2010; Bo et al., 2011; Reddy et al., 2009; Bhadra et al., 2009; Lai et al., 2012). It has a fairly good processability and conductivity, and from an economic point of view, it is significantly superior to other ICPs because its monomer, aniline, is less expensive than other monomers used for other ICPs. Its

\footnotetext{
*Author to whom correspondence should be addressed; email: epenriquez@ateneo.edu
} 
synthesis is very simple and its properties can be tuned easily (Kumar et al., Polyaniline, 2012; Bhadra et al., 2009).

Graphene, polyaniline, and graphene/ polyaniline composites have been used in different electronic applications, such as electrochemical electrodes in supercapacitors (Wu et al., Supercapacitors, 2010; Lai et al., 2012; Zhang et al., Graphene, 2010; Chen et al., 2012; Zhang and Zhao, 2012; Shen et al., 2013; Sarker and Hong, 2013; Liu et al., Three, 2013; Luo et al., 2013; Gao et al., 2012; Li et al., Fabrication, 2013; Jin et al., 2013; Shulga et al., 2013; Zhou et al., 2013), electroanalytical and bioelectroanalytical sensors (Lu et al., 2009; Bo et al., 2011; Shan et al., 2010; Chang et al., 2010; Bao et al., 2011; Sheng et al.; Huang et al., 2011; Liu et al., Novel, 2012; Li et al., Electrochemical, 2013; Radhapyari et al., 2013; Ruecha et al., 2014; Hu et al., 2013; Devi et al., 2013; Wu et al., Enhanced, 2013; Konwer et al., 2013), and organic/sensitized solar cells (Jung et al., 2011; Wan et al., 2011; Zhao et al., Polyaniline, 2009; Wang et al., 2012; Liu et al., Graphene, 2012; Chatterjee et al., 2013; Remyamol et al., 2013). However, most, if not all, graphene/polyaniline composites are blends-that is, the two are combined and reassembled through $\pi-\pi$ stacking. Although the properties of these composites are superior to either graphene or polyaniline, they are not robust. ICPs are known to be unstable due to their ease of oxidation. On the other hand, $\pi-\pi$ stacking, albeit strong as seen in the stacking of graphene sheets in graphite, is easily dislodged by exfoliation (Reddy et al., 2009). The dislodged polyaniline, without graphene, is prone to oxidation. Hence, it is highly plausible for graphene/polyaniline blends to quickly fail due to wear and tear caused by abrasion.

However, in situ graft polymerizations could be conducted with functionalized graphene as the substrate because different monomers could easily be immobilized, such as aniline (Reddy et al., 2009; Chatterjee et al., 2013; Remyamol et al., 2013; Mazur and Krysiński, 2001; Ren et al., 2012; Kumar et al., Synthesis,
2013). Unlike graphene/polyaniline blends, covalently-linked graphene/polyaniline composites would better withstand abrasion/ exfoliation because polyaniline is anchored to graphene. Covalently-linked graphene/ polyaniline composites have been synthesized recently through in situ graft polymerization of aniline that has been immobilized to graphene oxide through its functional groups (Kumar, et al., Polyaniline, 2012; Sahoo et al., 2012). However, the use of graphene oxide diminishes the potential of the composite, particularly its electronic properties vis-à-vis composites with pristine graphene.

In this study, graphene is functionalized with polyaniline by in situ graft polymerization of aniline in the presence of $p$-aminophenylfunctionalized graphene using ammonium peroxydisulfate as oxidizing agent. Covalentlylinked graphene/polyaniline was characterized and compared with graphene, polyaniline, and their physical blend using Raman, infrared and UV-Visible spectroscopy, and cyclic voltammetry.

\section{MATERIALS AND METHODS}

Materials. The solvents and reagents were used as purchased without further purification: graphite powder (Sigma-Aldrich), N,N-dimethylformamide (JT Baker), aniline (Fischer), $p$-nitroaniline, tin(II) chloride (JT Baker), sodium nitrite, hydrochloric acid, acetone (JT Baker), hydrogen peroxide (JT Baker), sulfuric acid. Deionized water (18 $\mathrm{m} \Omega-\mathrm{cm}$ ) was used for rinsing during washing.

Commercially available glass slides were used as substrates for Raman analysis.

Apparatus. Centrifugation was done using Hettich Zentrifugen Mikro 220 and Sorvall Legend RT Centrifuge. Sonication was done using GE 130 Ultrasonic Processor (Thomas Scientific) and Cole-Parmer 8891 Ultrasonic bath.

Synthesis of Graphene. Graphene was synthesized from graphite powder using microabrasive cleavage from a modified procedure of Hamilton et al. (2009). A 100-mg 
$(\sim 8.3 \mathrm{mmol})$ graphite powder was sonicated for 1 hour in $100 \mathrm{~mL} \quad N, N$-dimethylformamide (DMF), centrifuged at 4,600 rpm for $30 \mathrm{~min}$, and the resulting supernatant was collected. A $240-\mathrm{mL}$ graphene dispersion in DMF was filtered over a $0.45 \mu \mathrm{m}$ nylon membrane filter. The membrane filter was briefly sonicated in $30 \mathrm{~mL}$ DMF to dislodge graphene from it.

\section{Functionalization of p-Aminophenyl} (Aniline) Moiety in Graphene. Diazonium functionalization was conducted by filtering $240 \mathrm{~mL}$ DMF graphene and then redispersing it in $30 \mathrm{~mL} \mathrm{DMF}$ as a pre-concentration step to reduce the amount of solvent used for the reaction. The $240 \mathrm{~mL}$ DMF was used to exfoliate more graphene; this amount of DMF would be wasted if the reaction was conducted without pre-concentration. In addition, the functionalization of $p$-aminophenyl (aniline) moiety was conducted in two steps, namely: diazonium functionalization of $p$-nitrophenyl moiety from $p$-nitroaniline and then reduction of aryl nitro to aryl amino groups because $p$-phenylene-diamine (PPD), the reduced form of $p$-nitroaniline, cannot be used as both amino groups would have been available for diazotization.

The summary of steps is shown in a scheme in Figure 1. The residue from filtering $240 \mathrm{~mL}$ DMF graphene was re-sonicated in $30 \mathrm{~mL}$ DMF for $10 \mathrm{~min}$ at $40 \%$ amplitude. $0.26 \mathrm{~g} \quad(1.92 \mathrm{mmol}) \quad p$-nitroaniline and $0.160 \mathrm{~mL}(1.92 \mathrm{mmol}) \mathrm{HCl}$ was added and the mixture was purged with nitrogen gas for $10 \mathrm{~min}$ and pre-heated to $70{ }^{\circ} \mathrm{C} .0 .13 \mathrm{~g}$ $(1.92 \mathrm{mmol})$ sodium nitrite was added and the temperature was maintained for $1 \mathrm{~h}$ with constant agitation while protected from light. Water was added and the residue was collected by centrifugation at 4,600 rpm for $20 \mathrm{~min}$. The residue was washed with water and acetone until the supernatant was clear.

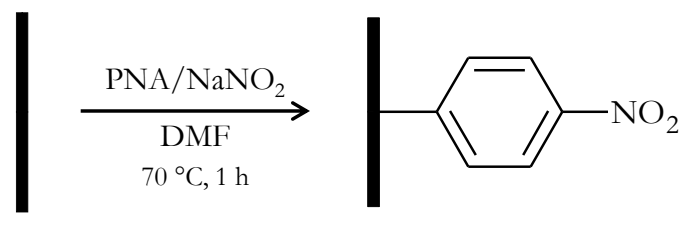

GNO

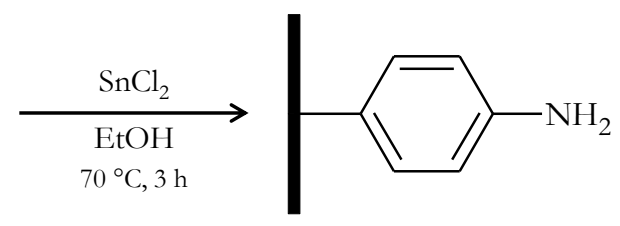

GNH

Figure 1. Functionalization of p-Aminophenyl (Aniline) Moiety in Graphene (represented by the dark line).

Reduction was done using a procedure adopted from Bellamy et al. (1984). $p$-Nitrophenyl-functionalized graphene (GNO) was sonicated in $30 \mathrm{~mL}$ ethanol for $10 \mathrm{~min}$ at $40 \%$ amplitude. $2.16 \mathrm{~g}(9.60 \mathrm{mmol})$ tin(II) chloride dihydrate was added and the mixture was heated to $70{ }^{\circ} \mathrm{C}$. The temperature was maintained for $3 \mathrm{~h}$ with constant agitation. The residue was collected by centrifugation at 4,600 rpm for $20 \mathrm{~min}$ and it was washed three times with ethanol and also with water and finally acetone.

In Situ Oxidative Graft Polymerization of Aniline. Here, $p$-Aminophenyl-functionalized graphene (GNH) was reacted in situ by oxidative graft polymerization of aniline using ammonium peroxydisulfate (APS) as oxidizing agent as summarized in Figure 2 .
p-Aminophenyl-functionalized graphene (GNH) was sonicated in $15 \mathrm{~mL}$ water for $10 \mathrm{~min}$ at $40 \%$ amplitude. $0.75 \mathrm{~mL}(8 \mathrm{mmol})$ hydrochloric acid was added to $0.75 \mathrm{~g}$ (8 mmol) aniline and dried under vacuum with slight heating. The resulting precipitate was added to the GNH dispersion and bubbled with nitrogen gas for at least $10 \mathrm{~min}$ in a septum-capped vessel. $2.28 \mathrm{~g}(10 \mathrm{mmol})$ ammonium peroxydisulfate was dissolved in $15 \mathrm{~mL}$ water and slowly introduced to the $\mathrm{GNH} /$ aniline mixture using a syringe while continuously bubbled with nitrogen. The resulting mixture was agitated at $120 \mathrm{rpm}$ for $6 \mathrm{~h}$ at $25{ }^{\circ} \mathrm{C}$ under nitrogen. The precipitate was collected by centrifugation at 4,600 rpm for $20 \mathrm{~min}$ and washed thrice with water and acetone. 


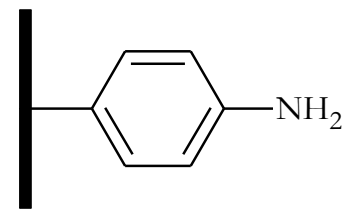

GNH

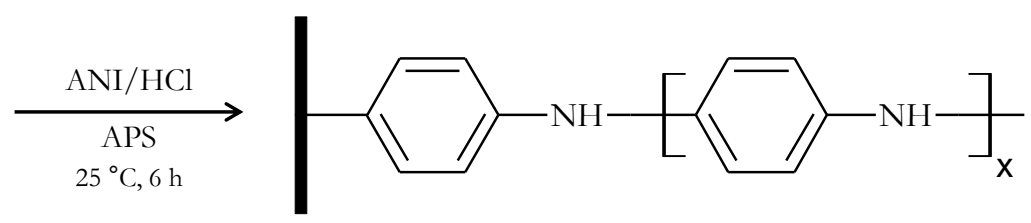

PANI-GNH

Figure 2. In situ Oxidative Graft Polymerization of Aniline to Graphene (represented by the dark line).

For comparison, a physical blend of polyaniline and graphene was prepared by substituting GNH with pristine graphene (Section 2.3). Polyaniline was prepared in the same way without graphene.

\section{Spectroscopic Characterization of Polyaniline/Graphene Composites. For} UV-Vis, the samples were dispersed by ultrasonication in ethanol for $10 \mathrm{~min}$ and centrifuged at $300 \mathrm{rpm}$ for $10 \mathrm{~min}$. The supernatants were collected and their corresponding absorption spectra were acquired in a quartz cuvette with a $0.5 \mathrm{~nm}$ scanning resolution using a Shimadzu UV-2401PC UV-Vis Recording Spectrophotometer.

For Raman studies, the exfoliated graphene flakes or modified exfoliated graphene flakes were dispersed in ethanol. The dispersion was then drop-cast onto 1 " $\times 0.5$ " glass slides and the solvent evaporated at room temperature. The Raman spectra were then analyzed using a home-built dispersive Raman confocal microspectroscope $(550 \mathrm{~mm}$ focal length, 1200 grooves/mm grating, excitation source of $514.5 \mathrm{~nm}$, and with a resolution better than $2 \mathrm{~cm}^{-1}$.) of the National Institute of Physics, UP-Diliman. Spectral analysis, which include baseline correction and normalization of the peaks relative to the most prominent carbon peak ( $G$ peak), were performed using Origin Pro 8 Peak Fit.

For FTIR, the samples were pelletized with $\mathrm{KBr}$ and run in transmittance mode at $4 \mathrm{~cm}^{-1}$ resolution using a Shimadzu IRAffinity-1; parameters used were: Happ-Ganzel apodization, at least 25 scans averaged, and baseline correction and single smoothing were done.
Electrochemical Characterization of Chemically Modified Graphene. The product was concentrated in the native solvent and drop-casted onto pre-treated pencil-lead graphite electrodes (PLGEs). Pretreatment consisted of a potential sweep from $-0.2 \mathrm{~V}$ to $1.0 \mathrm{~V}$ and back for 50 cycles with a sweep rate of $50 \mathrm{mV} / \mathrm{s}$ in a $1 \mathrm{M} \mathrm{HCl}$ solution using a three electrode cell. The prepared electrodes were then left overnight to dry. The electrodes were then attached to a Metrohm Autolab $\mu$ AutolabIII/FRA2 in a three-electrode configuration with a silver/silver chloride reference electrode and a platinum counter electrode. Two different electrolytes were used, $0.1 \mathrm{M} \mathrm{KCl}$ with $1 \mathrm{mM}$ $\mathrm{K}_{3} \mathrm{Fe}(\mathrm{CN})_{6}$ and $1 \quad \mathrm{M} \quad \mathrm{H}_{2} \mathrm{SO}_{4}$. Cyclic voltammetry $(\mathrm{CV})$ was performed within a potential range of -0.2 to $1.0 \mathrm{~V}$ with varying sweep rates for 10 cycles each.

The triethylamine, THF and diethyl ether used in the synthesis of the salicylaldehyde precursor were freshly distilled prior to use. All other starting materials and solvents were purchased commercially and used as received. Proton NMR spectra and infrared (IR) spectra were measured using a $500 \mathrm{MHz}$ JNM-LA500 and a Shimadzu FTIR-8300, respectively. UVVis spectra were recorded on a Shimadzu MultiSpec-1500 at room temperature with $\mathrm{CH}_{2} \mathrm{Cl}_{2}$ as solvent. For low temperature UVVis measurements, an Alpha Engineering Ltd. CS-96-2 cryostat and Model TC 22-A thermo controller were used. High resolution and electron-ionization mass spectrometric measurements were carried out on a JEOL JMS-SX102A. For the complex, fast atomic bombardment (FAB) with p-nitro-benzyl alcohol (NBA) as matrix was utilized. Elemental analyses were obtained with the use of a Perkin-Elmer 2400-II and magnetic susceptibility measurements were done by way 
of the Faraday method using a CAHN 1000 Electrobalance and $\mathrm{Hg}\left[\mathrm{Co}(\mathrm{SCN})_{4}\right]$ as reference compound.

\section{RESULTS AND DISCUSSION}

Functionalization of p-Aminophenyl (Aniline) Moiety in Graphene. GNO and GNH (Figure 1) were characterized vis-à-vis pristine graphene (PG) using FTIR and Raman spectroscopy to confirm successful functionalization. The spectrum of exfoliated graphene (PG) hardly have any features indicating the absence of functional groups visible in the IR spectrum (Figure 3). This contrasts with the FTIR spectrum of the starting material used by Kumar et al. (2012) in which graphite oxide (GO) was used. The spectra of GNO and GNH in this region have peaks corresponding to the functional groups (bending vibrations for $\mathrm{N}-\mathrm{H}, \mathrm{N}-\mathrm{O}$ and $\mathrm{C}-\mathrm{N}$ ) introduced; e.g. for the GNH sample, a more prominent peak at $1620 \mathrm{~cm}^{-1}$ typically attributed to the $-\mathrm{NH}_{2}$ scissoring vibration is present. These results are indicative of successful modification of graphene. However, peaks at $2840 \mathrm{~cm}^{-1}$ and $2900 \mathrm{~cm}^{-1}$, which correspond to aliphatic $\mathrm{C}-\mathrm{H}$ stretch, cannot be accounted for in the structures of GNO and GNH while the peak at $\sim 3400 \mathrm{~cm}^{-1}$, which correspond to $\mathrm{O}-\mathrm{H}$ or $\mathrm{N}-\mathrm{H}$ stretch, cannot be accounted for that of GNO.

Furthermore, the unmodified and modified graphenes have different dispersibilities in ethanol. GNO in ethanol remains dark after centrifugation whereas GNH easily settles down. On the other hand, in water, GNH is easier to disperse as fine particles whereas PG hardly breaks up and easily agglomerates when allowed to stand. This difference in dispersibility lies in the nitro and amino groups present: nitro and amino groups are polar, so they favorably interact with ethanol; however, amino groups are easily ionizable and the presence of these charges make them less dispersible in ethanol which is only weakly polar compared with water. In summary, their respective dispersibilities in ethanol are in the order of $\mathrm{GNO}>>\mathrm{PG}>\mathrm{GNH}$.

Though the FTIR data confirmed the presence of the nitro and amino functional groups, it could not differentiate between covalent linkage and non-covalent interaction between graphene and the moieties. Washing

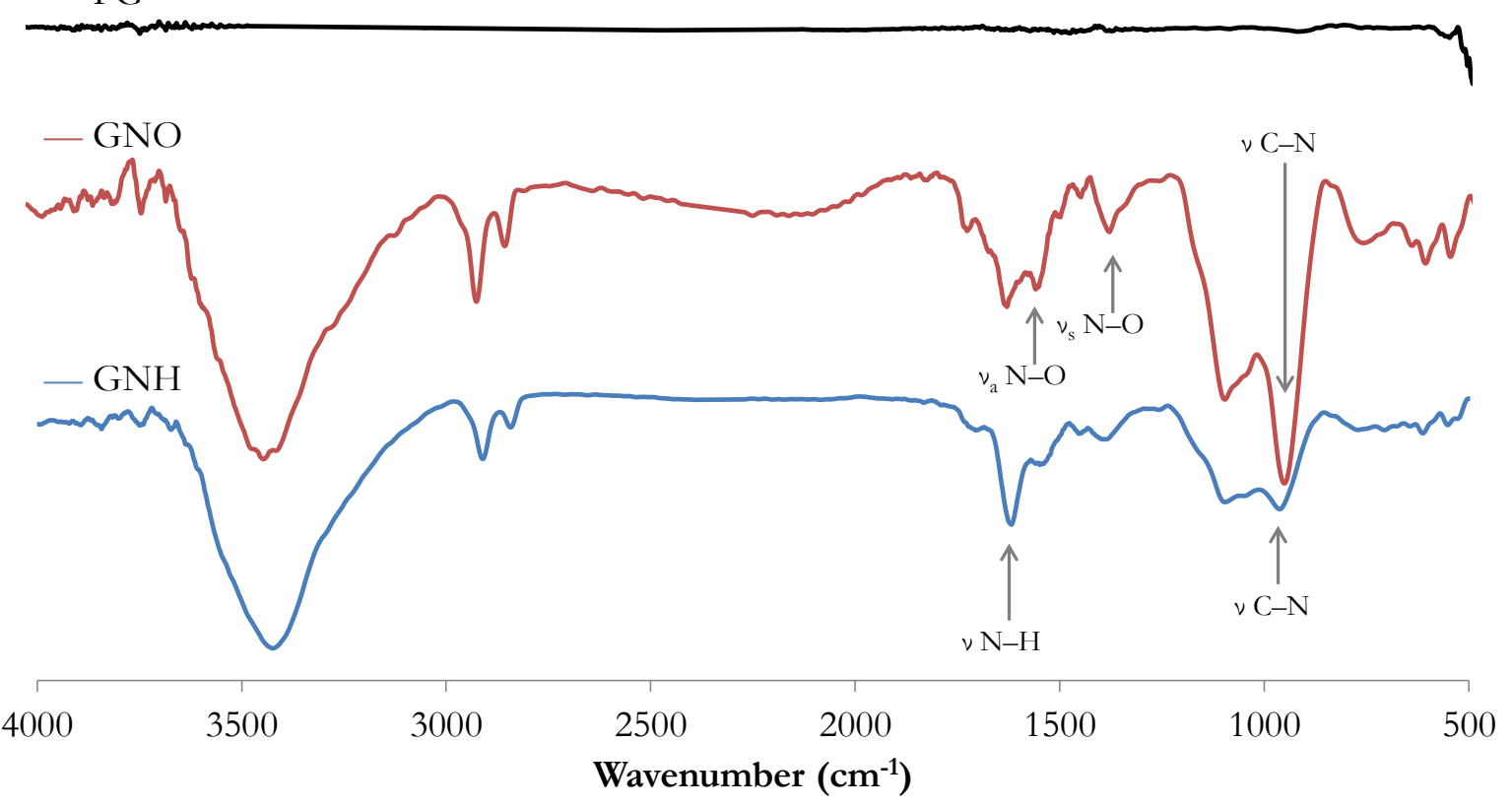

Figure 3. FTIR spectra of graphene (PG), p-nitrophenyl-functionalized graphene (GNO) and p-aminophenylfunctionalized graphene (GNH). 
alone may not have removed residual aminocontaining reagents. Thus, Raman spectroscopy was used to confirm covalent functionalization as this is able to detect a change in the hybridization of the carbon in graphene, from $s p^{2}$ to $s p^{3}$, including when it forms a covalent bond with a functional moiety. At the excitation of $514.5 \mathrm{~nm}$, diamond-like carbons produce a Raman shift at $\sim 1350 \mathrm{~cm}^{-1}$, denoted as the D peak, while graphitic carbons have a Raman shift at $\sim 1580 \mathrm{~cm}^{-1}$, the $G$ peak. These two shifts, respectively, are correlated to the $s p^{3}$ and $s p^{2}$ carbons present in carbonaceous material, with the former resulting from symmetrybreaking effects (Jorio, 2011). The G peak $\left(\sim 1580 \mathrm{~cm}^{-1}\right)$, which corresponds to $s p^{2}$ carbon-carbon bond vibration, remains largely unaffected by chemical reactions in the absence of resonance enhancement or formal charging (Sharma R et al., 2010). The D peak at $\sim 1350 \mathrm{~cm}^{-1}$ corresponds to the $s p^{3}$ carbon vibrational modes and is indicative of defects in the graphene structure. The $\mathrm{D} / \mathrm{G}$ peak ratio is usually used to monitor the extent of hybridization, and therefore extent of reaction or functionalization, where the $G$ peak can serve as reference peak (Hsiao et al., 2010).

The Raman spectra for the samples are compared with the exfoliated graphene in Figure 4. Here, we note that the D peak is present in exfoliated graphene as these indicate defect areas particularly at the edges of the graphene flakes. We also note a major difference in these samples compared with the work by Kumar et al. (2012) — the Raman peaks in our samples are sharper where the $D$ and $G$ peaks are non-overlapping, with $G$ still more prominent than the D peak, compared with the broader, overlapping bands in Kumar's work. This is indicative of less disruption of the graphene structure in our scheme of direct functionalization of graphene which did not pass through the graphene oxide step of Kumar et al.

The $D / G$ peak ratios are summarized in Table 1. The values for the modified graphene are much higher compared to PG: 2.5 times for GNO and 3.4 times for GNH.

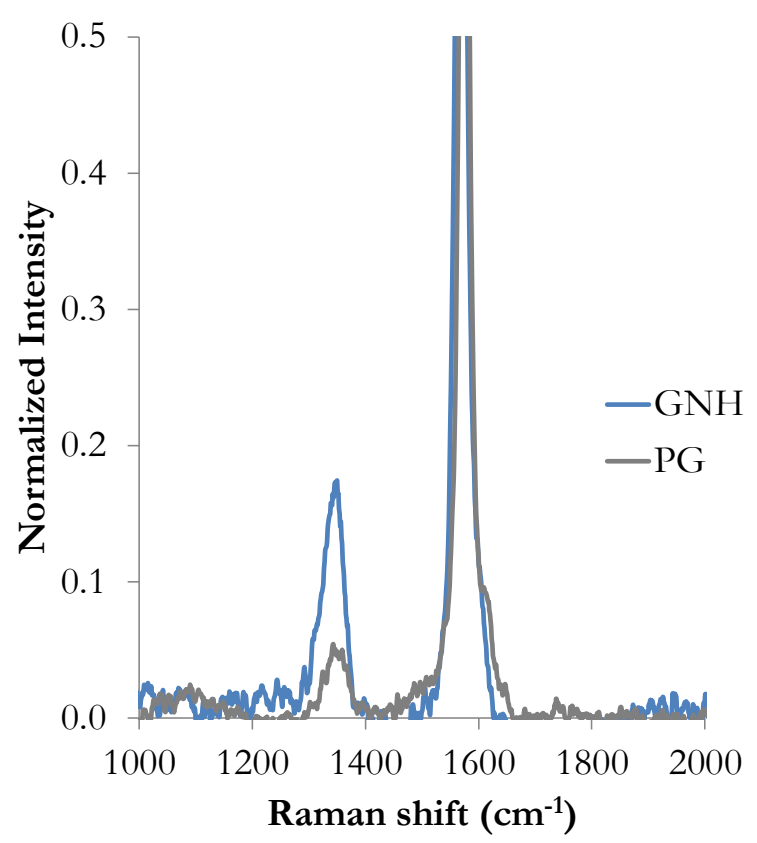

Figure 4. Normalized (Intensity of $G$ peak at $\sim 1580 \mathrm{~cm}^{-1}$ set to 1.0) Raman spectra of graphene (PG), pnitrophenyl-functionalized graphene (GNO) and p-aminophenyl-functionalized graphene (GNH). The prominent $G$ peak is truncated to reveal the $D$ peak. 
Table 1. Intensites of the Raman D and G Peaks, and the D/G Ratios of graphene (PG), p-nitrophenyl-functionalized graphene (GNO) and p-aminophenylfunctionalized graphene (GNH).

\begin{tabular}{cccc}
\hline Sample & $\begin{array}{c}\text { D peak } \\
\left(\begin{array}{c}\mathbf{1 3 5 0} \\
\left.\mathbf{c m}^{-1}\right)\end{array}\right.\end{array}$ & $\begin{array}{c}\text { G peak } \\
(\mathbf{\sim 1 5 8 0} \\
\left.\mathbf{c m}^{-1}\right)\end{array}$ & $\begin{array}{c}\text { D/G } \\
\text { Ratio }\end{array}$ \\
\hline PG & 0.0638 & 1.00 & 0.0638 \\
\hline GNO & 0.1757 & 1.00 & 0.1757 \\
\hline GNH & 0.2374 & 1.00 & 0.2374 \\
\hline
\end{tabular}

This implies structural modification of the carbon network of graphene, which arises from the change in hybridization of the affected carbon atoms, from $s p^{2}$ to $s p^{3}$. This conforms with the FTIR results and further confirms successful covalent functionalization of presumably $p$-nitrophenyl and $p$-aminophenyl moieties on graphene. It is expected that the $\mathrm{D} / \mathrm{G}$ ratios for the $\mathrm{GNO}$ and $\mathrm{GNH}$ should be the same because the reduction of the nitro groups in GNO does not affect the graphene-phenyl bond. However, the $\mathrm{D} / \mathrm{G}$ ratios are somewhat different. This may possibly be due to the difference in the effect of scattering of the functional groups but may also be caused by the polydispersity of the sizes of the exfoliated flake samples. Polydispersity arises from solution phase techniques used to produce graphene dispersions (Green and Hersam, 2010). Single graphene layers seem to be more reactive than bi- or multilayers under Raman spectroscopy because the process samples the inner carbon atoms which are shielded from chemical treatment; the $G$ peak intensity increases for a given $\mathrm{D}$ peak caused by the functionalization of the outer layers for multi-layered graphene. Also, as previously reported, there is preferential functionalization of the edges of graphene over the bulk. An altered electronic structure is expected at the edges compared to the bulk region due to symmetry breaking of the honeycomb lattice at the edges. In addition, the dangling bonds at the edges can also be a major contribution to the enhanced edge reactivity (Sharma R et al., 2010).
The efficiency/yields of these two processes cannot be reliably quantified. FTIR was used to confirm the presence of nitro and amino groups in the sample; however, the peaks cannot be used to quantify the functional groups present due to the lack of an internal standard. In addition, peaks that differentiate the two samples overlap, so it is impossible to distinguish the amino groups from nitro groups in GNH if it had undergone incomplete reduction. On the other hand, Raman spectroscopy was used to confirm the covalent functionalization of graphene with $p$-substituted phenyl moieties but the polydispersity of the exfoliated graphene flakes makes it difficult to quantify the exact yield. Further studies are needed to quantify the yield of these two processes.

\section{In Situ Oxidative Graft Polymerization of} Aniline. FTIR spectroscopy confirmed the successful synthesis of polyaniline and its presence in either the physical blend of PANI with graphene (PANI-G) and in the covalently functionalized PANI-grafted-G (PANI-GNH) composites showing the typical bands for PANI (Figure 5). The broad peak around $3430 \mathrm{~cm}^{-1}$ appears due to the $\mathrm{N}-\mathrm{H}$ stretch in PANI. The peaks around $1560 \mathrm{~cm}^{-1}$ and $1480 \mathrm{~cm}^{-1}$ are due to the $\mathrm{C}=\mathrm{C}$ stretching deformations of the quinonoid and benzenoid structures of PANI, respectively. The characteristic peak around $1120 \mathrm{~cm}^{-1}$ is due to the N-Q-N-Q stretch of the quinonoid ring. Only the blend (PANI/G) showed extra bands around $1750 \mathrm{~cm}^{-1}$ (possibly carbonyl groups at the edges of graphene) and $950 \mathrm{~cm}^{-1}$ (Yelil Arasia et al., 2009; Wang et al., Hierarchical, 2013). The FTIR spectra are similar to those of PANI and graphene/polyaniline composites reported. (Yelil Arasia et al., 2009; Ni et al., 2014). However, FTIR spectroscopy could not differentiate the two types of composites.

Nonetheless, the general properties of the two polyaniline-graphene composites differ from each other. Right after polymerization, PANI/G precipitates were large and loose, did not break up into smaller particles in water when agitated, and there were green 


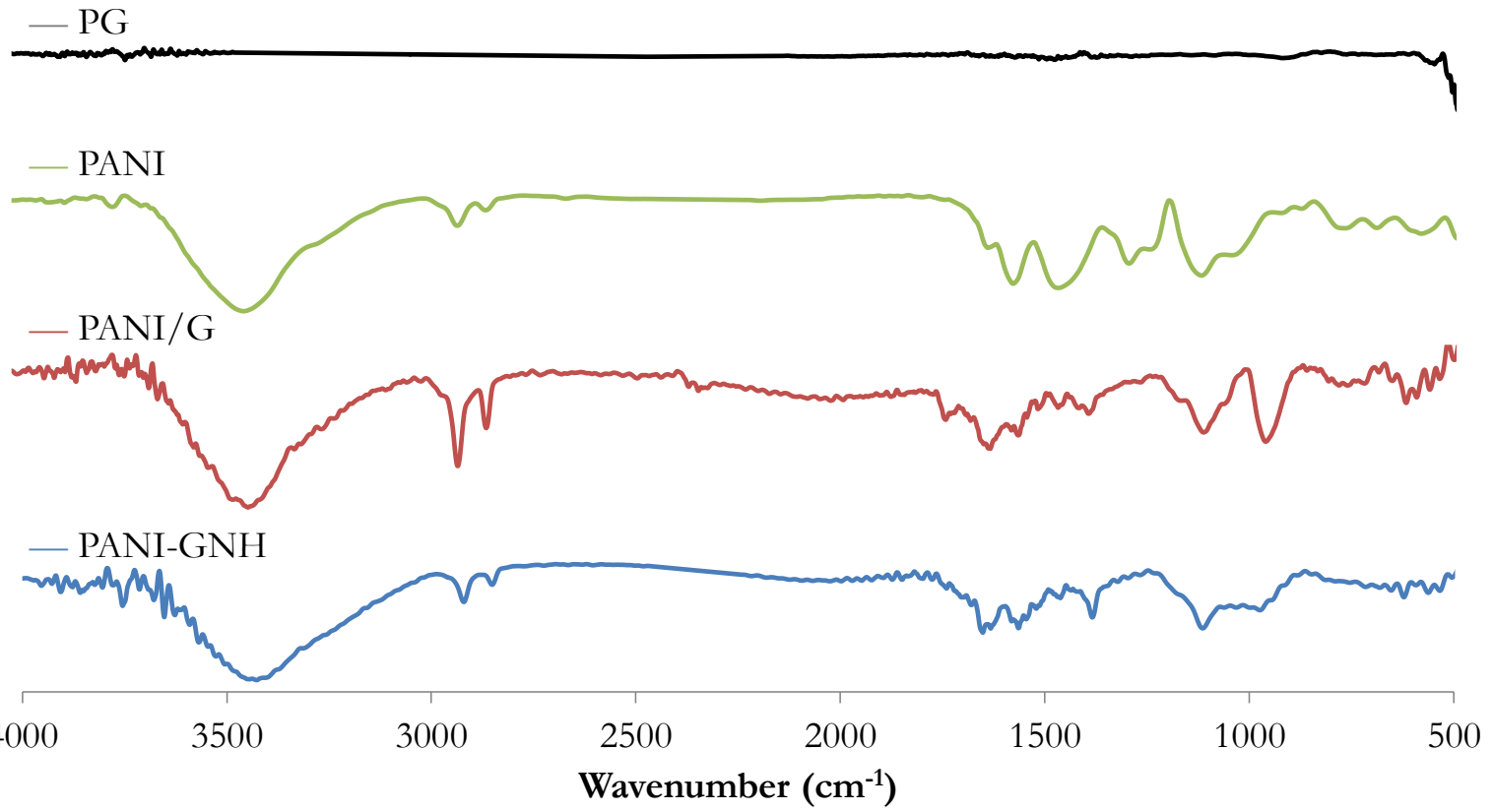

Figure 5. FTIR spectra of graphene (PG), polyaniline (PANI) and polyaniline+graphene blend (PANI/G) and the grafted case (PANI-GNH).

precipitates of polyaniline. On the other hand PANI-GNH precipitates clumped together but were easily broken down into smaller particles in water when agitated, and are uniformly black to dark green. Upon drying, optical microscope images of PANI/G precipitates retained the appearance of graphene and polyaniline with the presence of loose graphene sheets (light gray). In comparison, PANI-GNH precipitates clumped together (Figure 6). This difference in behavior in water is indicative of a different type of linkage (physical versus chemical) for these composites. However, gross morphology is not a direct proof of structure.

UV-Visible spectroscopy further reveals the difference between the two composites, and compared with PG or PANI alone (Figure 7). The peak at $270 \mathrm{~nm}$ in graphene, PANI/G and PANI-GNH confirms the presence of graphene (Shan et al., 2010) while the absence of a peak at $230 \mathrm{~nm}$ confirms the absence of graphene oxide. The absorption band around

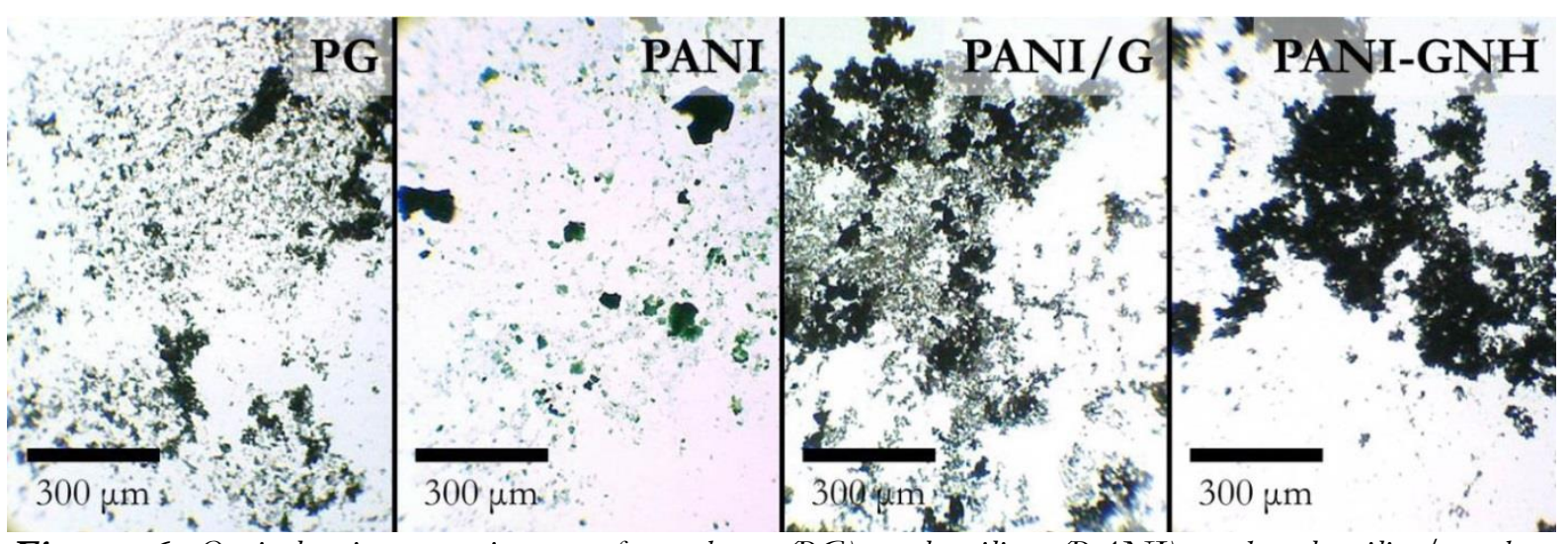

Figure 6. Optical microscopy images of graphene (PG), polyaniline (PANI) and polyaniline/graphene composites (PANI/G and PANI-GNH). 


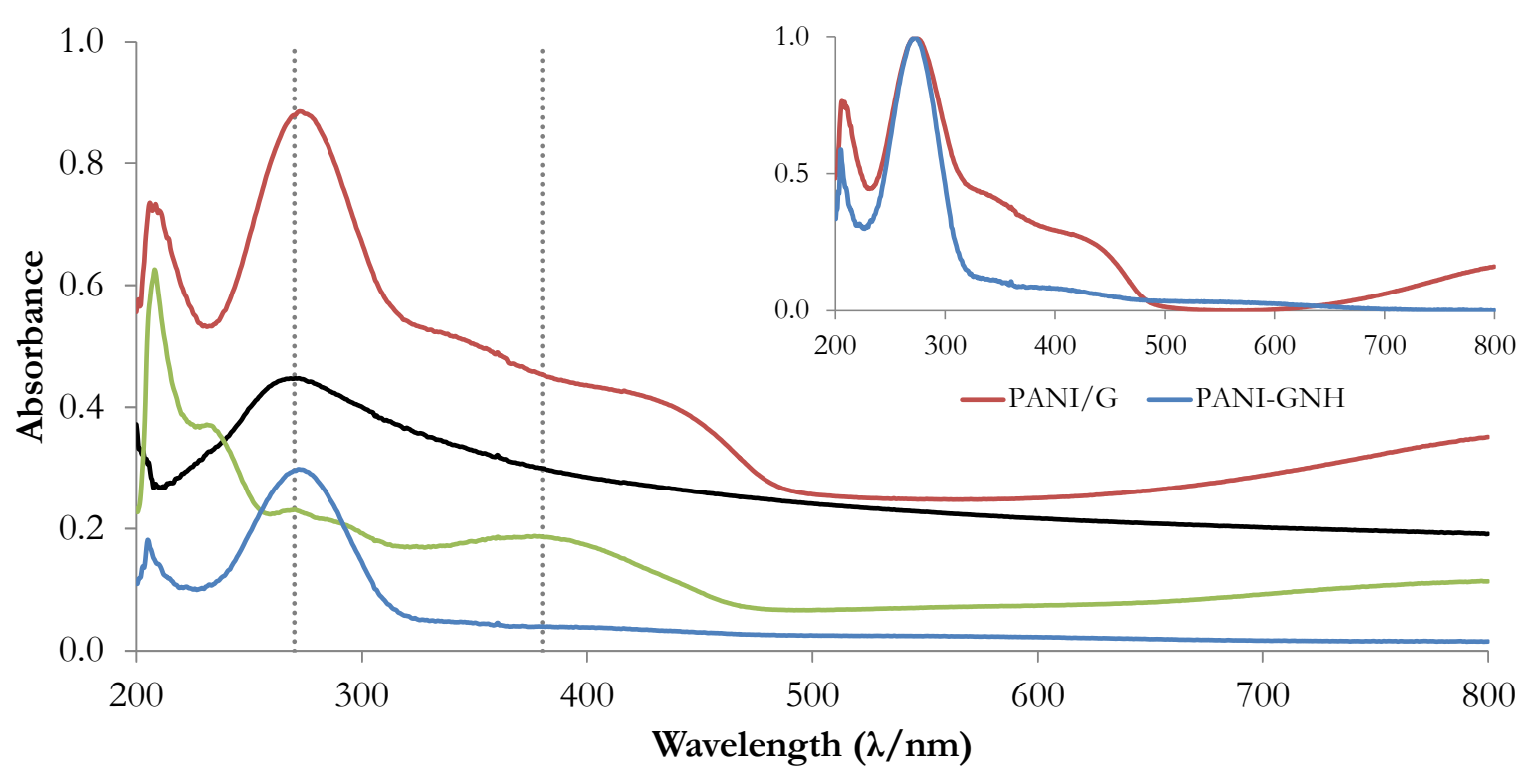

$-\mathrm{PG}-\mathrm{PANI}-\mathrm{PANI} / \mathrm{G}-\mathrm{PANI}-\mathrm{GNH}$

Figure 7. UV-Visible spectra of graphene (PG), polyaniline (PANI) and polyaniline/graphene composites (PANI/G and PANI-GNH) in ethanol. Inset: comparison of polyaniline composites (PANI/G and $P A N I-G N H)$ normalized at $270 \mathrm{~nm}$.

$380 \mathrm{~nm}$ for PANI appears to have a bathochromic shift in the two composites which may be due to the interaction between polyaniline and graphene. This interaction, mainly due to $\pi$ - $\pi$ stacking, stabilizes the $\pi \rightarrow$ $\pi^{*}$ transition at 320-380 nm (Saini and Basu, 2012) in polyaniline, which resulted in a corresponding bathochromic shift (Sharma and Kumar, 2010). It is notable that the graphene and PANI optical properties are retained in the case of the grafted composite (PANI-GNH) - dominated mostly by the graphene band, with small PANI contribution. The relatively low PANI absorbance in PANI-GNH indicates low density of functionalization per flake. Further studies are needed to quantify the extent of functionalization of PANI relative to GNH.

The characterization methods used to analyze the two graphene/polyaniline composites cannot be used to quantify the extent of polymerization of aniline relative to graphene. This is due to the lack of an internal standard in the FTIR. On the other hand, the differences in the dispersibility of graphene, polyaniline and graphene/polyaniline composites in a solvent transparent to UV-Vis and the tendency of the samples to scatter light makes quantification using BeerLambert's Law inconclusive. Further studies are needed to quantify the extent of functionalization of PANI relative to GNH.

Electrochemical Characterization. The electrochemical properties of the graphene composites were compared alongside with PG and PANI alone, using each of them as working electrode (coated on graphite lead) and using potassium hexacyanidoferrate(III) as internal reference redox couple. The cyclic voltammograms are shown in Figure 8. The redox peaks of the potassium hexacyanidoferrate(III) is seen for the PG electrode with an additional irreversible reduction peak appearing at around $+0.82 \mathrm{~V}$ (vs $\mathrm{Ag} \mid \mathrm{AgCl}$ ) (Bo et al., 2011), which may be attributed to the reduction of the native oxidized carbon at the edges of the graphene flakes. These CV peaks were swamped by the PANI redox bands in the case of PANI/G or PANI-GNH (compare with PANI for example). There is slight shifting of the peak positions but overall they are similar for the 


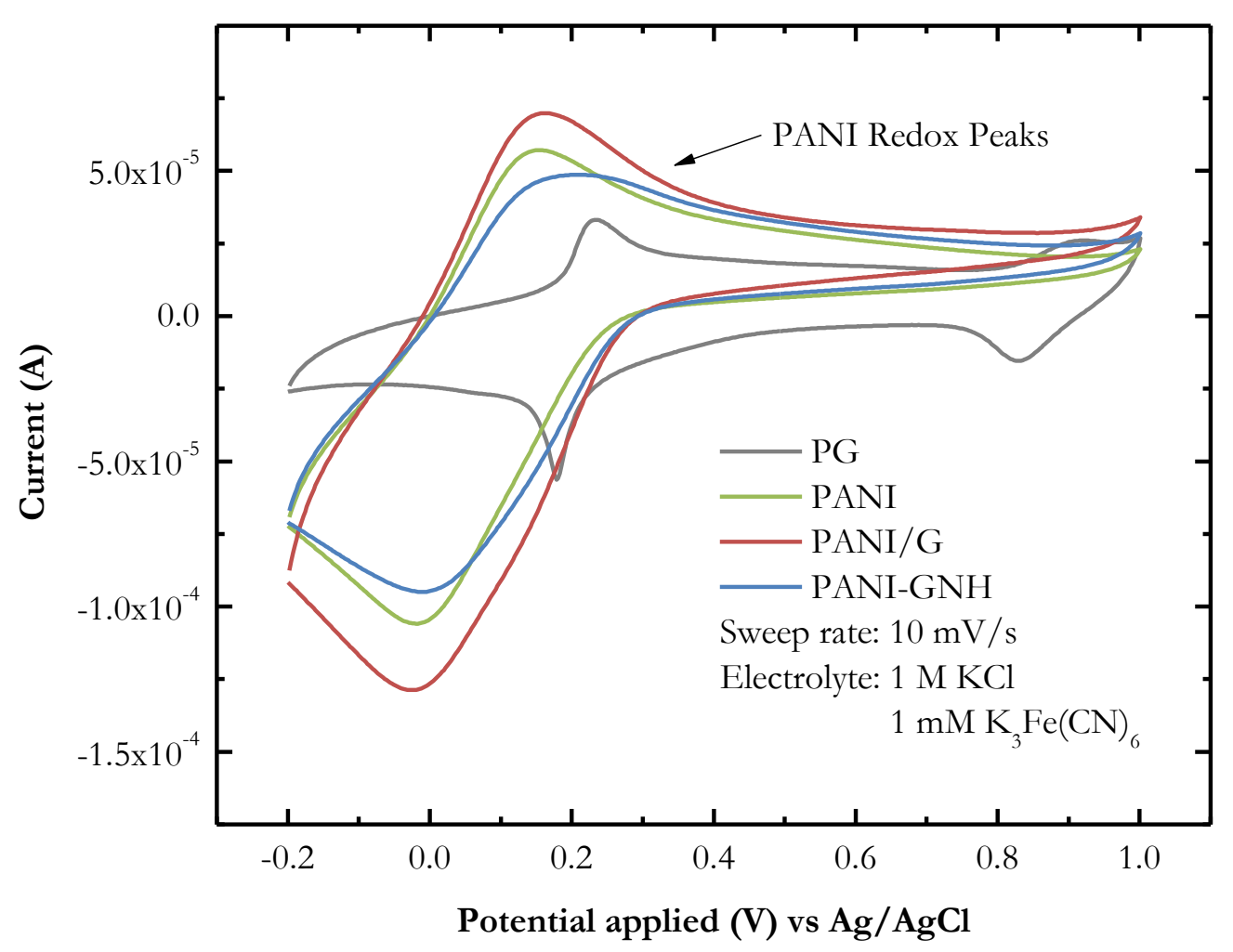

Figure 8. CV scans of graphene (PG), polyaniline (PANI), graphene /polyaniline blend (PANI/G), and covalently-linked graphene/polyaniline (PANI-GNH).

two composites. This indicates that PANI remains electroactive and dominates the property even when grafted to graphene.

\section{CONCLUSION}

Polyaniline in situ grafted to graphene was successfully synthesized. The process did not go through the usual graphite oxide intermediate to produce a PANI-GNH covalent composite. This composite was compared vis-à-vis polyaniline/graphene blend showing similar FTIR spectra, different UV-Vis absorption spectra indicating possibly low grafting density, and similar electrochemical property. Compared with the physical blend of PANI and graphene, the PANI-GNH is predicted to be more robust because of the covalent link. Additional characterization and studies are needed to evaluate the extent of grafting and subtle changes in the PANI and/or graphene properties.

\section{ACKNOWLEDGEMENT}

This work is done in part with funding support from the Philippine Council for Industry, Energy, and Emerging Technology Research and Development of the Department of Science and Technology (PCIEERD-DOST). EPE acknowledges the Chevron Philippines, Inc. Endowed Professorial Chair in the Ateneo. The Commission on Higher Education (CHED) is also acknowledged for the KIMIKA Writing Workshop held last 10-12 April 2013.

\section{REFERENCES}

Bao Y, Song J, Mao Y, Han D, Yang F, Niu L, et al. Graphene Oxide-Templated Polyaniline Microsheets toward Simultaneous Electrochemical Determination of AA/DA/ UA. Electroanalysis. 2011; 23(4):878-84.

Bellamy FD, Ou K. Selective reduction of aromatic nitro compounds with stannous chloride in non acidic and non aqueous medium. Tetrahedron Lett. 1984; 25(8):839-42. 
Bhadra S, Khastgir D, Singha NK, Lee JH. Progress in preparation, processing and applications of polyaniline. Prog Polym Sci. 2009 Aug; 34(8):783-810.

Bo Y, Yang H, Hu Y, Yao T, Huang S. A novel electrochemical DNA biosensor based on graphene and polyaniline nanowires. Electrochimica Acta. 2011 Feb 15; 56(6):267681.

Chang Yanhong, Wang Bin, Luo Hui, Zhi Linjie. Synthesis of Polyaniline/Graphene Composites and Its Application in Detecting Heavy Metal Ions. E-Prod E-Serv E-Entertain ICEEE 2010 Int Conf. 2010. p. 1-4.

Chatterjee S, Layek RK, Nandi AK. Changing the morphology of polyaniline from a nanotube to a flat rectangular nanopipe by polymerizing in the presence of aminofunctionalized reduced graphene oxide and its resulting increase in photocurrent. Carbon. 2013 Feb; 52(0):509-19.

Chen F, Liu P, Zhao Q. Well-defined graphene/polyaniline flake composites for high performance supercapacitors. Electrochimica Acta. 2012 Aug 1; 76(0):62-8.

Devi R, Relhan S, Pundir CS. Construction of a chitosan/polyaniline/graphene oxide nanoparticles/polypyrrole/Au electrode for amperometric determination of urinary/plasma oxalate. Sensors Actuators B Chem. 2013 Sep; 186(0):17-26.

Dimitriev OP. Confinement of Polyaniline Chains in Thin Layers of the Polymer Solution. J Colloid Interface Sci. 2001; 235:380-382.

Gao Z, Yang W, Wang J, Wang B, Li Z, Liu Q, et al. A New Partially Reduced Graphene Oxide Nanosheet/Polyaniline Nanowafer Hybrid as Supercapacitor Electrode Material. Energy Fuels. 2012 Dec 15; 27(1):568-75.

Green AA, Hersam MC. Emerging Methods for Producing Monodisperse Graphene Dispersions. J Phys Chem Lett. 2010; 1(2):544-549.
Hamilton CE, Lomeda JR, Sun Z, Tour JM, Barron AR. High-Yield Organic Dispersions of Unfunctionalized Graphene. Nano Lett. 2009 Oct 14; 9(10):3460-2.

Hsiao MC, Liao SH, Yen MY, Liu PI, Pu NW, Wang CA, Ma CCM. Preparation of covalently functionalized graphene using residual oxygen-containing functional groups. ACS App Mater Inter. 2010; 2(11): 3092-9.

Hu X-W, Mao C-J, Song J-M, Niu H-L, Zhang $\mathrm{S}-\mathrm{Y}$, Huang $\mathrm{H}$. Fabrication of GO/PANi/ CdSe nanocomposites for sensitive electrochemiluminescence biosensor. Biosens Bioelectron. 2013 Mar 15; 41(0):372-8.

Huang J, Lin Q, Zhang X, He X, Xing X, Lian $\mathrm{W}$, et al. Electrochemical immunosensor based on polyaniline/poly (acrylic acid) and Au-hybrid graphene nanocomposite for sensitivity enhanced detection of salbutamol. Food Res Int. 2011 Jan; 44(1):92-7.

Jin Y, Huang S, Zhang M, Jia M. Preparation of sulfonated graphene-polyaniline nanofiber composites by oil/water interfacial polymerization and their application for supercapacitors. Synth Met. 2013 Mar 15; 168(0):58-64.

Jorio A, Dresselhaus M, Saito R, Dresselhaus GF. Raman Spectroscopy in Graphene Related Systems. 2011, Weinheim: Wiley-VCH Verlag GmbH \& Co. KGaA, 91.

Jung JW, Lee JU, Jo WH. High-Efficiency Polymer Solar Cells with Water-Soluble and Self-Doped Conducting Polyaniline Graft Copolymer as Hole Transport Layer. J Phys Chem C. 2010 Jan 14; 114(1):633-7.

Konwer S, Guha A, Dolui S. Graphene oxidefilled conducting polyaniline composites as methanol-sensing materials. J Mater Sci. 2013 Feb 1; 48(4):1729-39.

Kumar M, Singh K, Dhawan SK, Tharanikkarasu K, Chung JS, Kong B-S, et al. Synthesis and characterization of covalentlygrafted graphene-polyaniline nanocomposites 
and its use in a supercapacitor. Chem Eng J. 2013 Sep; 231(0):397-405.

Kumar NA, Choi H-J, Shin YR, Chang DW, Dai L, Baek J-B. Polyaniline-Grafted Reduced Graphene Oxide for Efficient Electrochemical Supercapacitors. ACS Nano. 2012 Jan 25; 6(2):1715-23.

Lai L, Yang H, Wang L, Teh BK, Zhong J, Chou H, et al. Preparation of Supercapacitor Electrodes through Selection of Graphene Surface Functionalities. ACS Nano. 2012 May 26; 6(7):5941-51.

Li C, Shi G. Synthesis and electrochemical applications of the composites of conducting polymers and chemically converted graphene. Electrochimica Acta [Internet]. In Press, Corrected Proof. Available from: http://www.sciencedirect.com/science/article /B6TG0-51WV07S-2/2/529dac0056159b3a9 df4d2308e4c665e

Li J, Liu S, Yu J, Lian W, Cui M, Xu W, et al. Electrochemical immunosensor based on graphene-polyaniline composites and carboxylated graphene oxide for estradiol detection. Sensors Actuators B Chem. 2013 Nov; 188(0):99-105.

Li Y, Peng H, Li G, Chen K. Synthesis and electrochemical performance of sandwich-like polyaniline/graphene composite nanosheets. Eur Polym J. 2012 Aug; 48(8):1406-12.

Li Z-F, Zhang H, Liu Q, Sun L, Stanciu L, Xie J. Fabrication of High-Surface-Area Graphene/Polyaniline Nanocomposites and Their Application in Supercapacitors. ACS Appl Mater Interfaces. 2013 Mar 12; 5(7):2685-91.

Liu C-Y, Huang K-C, Chung P-H, Wang C-C, Chen C-Y, Vittal R, et al. Graphene-modified polyaniline as the catalyst material for the counter electrode of a dye-sensitized solar cell. J Power Sources. 2012 Nov 1; 217:152-7.

Liu H, Wang Y, Gou X, Qi T, Yang J, Ding Y. Three-dimensional graphene/polyaniline composite material for high-performance supercapacitor applications. Mater Sci Eng B. 2013 Mar 20; 178(5):293-8.

Liu L, Ryu S, Tomasik MR, Stolyarova E, Jung N, Hybertsen MS, et al. Graphene Oxidation: Thickness-Dependent Etching and Strong Chemical Doping. Nano Lett. 2008 Jul 1; 8(7):1965-70.

Liu S, Xing X, Yu J, Lian W, Li J, Cui M, et al. A novel label-free electrochemical aptasensor based on graphene-polyaniline composite film for dopamine determination. Biosens Bioelectron. 2012 Jun; 36(1):186-91.

Lu C-H, Yang H-H, Zhu C-L, Chen X, Chen G-N. A Graphene Platform for Sensing Biomolecules. Angew Chem Int Ed. 2009; 48(26):4785-7.

Luo Z, Zhu L, Zhang H, Tang H. Polyaniline uniformly coated on graphene oxide sheets as supercapacitor material with improved capacitive properties. Mater Chem Phys. 2013 May 15; 139(2-3):572-9.

Mazur M, Krysiński P. Covalently Immobilized 1,4-Phenylenediamine on 11Mercaptoundecanoic Acid-Coated Gold: Effect of Surface-Confined Monomers on the Chemical in Situ Deposition of Polyaniline and Its Derivatives. Langmuir. 2001 Oct 1; 17(22):7093-101.

Ni SB, Li HB, Li S, Zhu JL, Tan J Sun XY, et al. Low-voltage blue-phase liquid crystals with polyaniline-functionalized graphene nanosheets. J Mater Chem C. 2014; 2:1730-1735.

Radhapyari K, Kotoky P, Das MR, Khan R. Graphene-polyaniline nanocomposite based biosensor for detection of antimalarial drug artesunate in pharmaceutical formulation and biological fluids. Talanta. 2013 Jul 15; 111(0):47-53.

Reddy KR, Sin BC, Ryu KS, Kim J-C, Chung $\mathrm{H}$, Lee Y. Conducting polymer functionalized multi-walled carbon nanotubes with noble metal nanoparticles: Synthesis, morphological characteristics and electrical properties. Synth Met. 2009 Apr; 159(7-8):595-603. 
Remyamol T, John H, Gopinath P. Synthesis and nonlinear optical properties of reduced graphene oxide covalently functionalized with polyaniline. Carbon. 2013 Aug; 59(0):308-14.

Ren L, Huang S, Zhang C, Wang R, Tjiu W, Liu T. Functionalization of graphene and grafting of temperature-responsive surfaces from graphene by ATRP "on water."J Nanoparticle Res. 2012 Jun 1; 14(6):1-9.

Ruecha N, Rangkupan R, Rodthongkum N, Chailapakul O. Novel paper-based cholesterol biosensor using graphene/polyvinylpyrrolidone/polyaniline nanocomposite. Biosens Bioelectron. 2014 Feb 15; 52(0):13-9.

Sahoo S, Karthikeyan G, Nayak G, Das C. Modified nanocomposites for supercapacitor application. Macromol Res. 2012 Apr 1; 20(4):415-21.

Saini D, Basu T. Synthesis and characterization of nanocomposites based on polyaniline-gold/grapheme nanosheets. Appl Nanosci. 2012; 2:467-479.

Sarker AK, Hong J-D. Electrochemical reduction of ultrathin graphene oxide/polyaniline films for supercapacitor electrodes with a high specific capacitance. Colloids Surfaces Physicochem Eng Asp. 2013 Sep 5; 436(0):967-74.

Shan C, Yang H, Han D, Zhang Q, Ivaska A, Niu L. Electrochemical determination of $\mathrm{NADH}$ and ethanol based on ionic liquidfunctionalized graphene. Biosens Bioelectron. 2010 Feb 15; 25(6):1504-8.

Sharma R, Baik JH, Perera CJ, Strano MS. Anomalously Large Reactivity of Single Graphene Layers and Edges toward Electron Transfer Chemistries. Nano Lett. 2010 Feb 10; 10(2):398-405.

Sharma S, Kumar D. Study on solvatochromic behaviour of polyaniline and alkyl substituted polyanilines. Indian J Eng Mater S. 2010; 17:231-237.
Shen J, Yang C, Li X, Wang G. HighPerformance Asymmetric Supercapacitor Based on Nanoarchitectured Polyaniline/Graphene/Carbon Nanotube and Activated Graphene Electrodes. ACS Appl Mater Interfaces. 2013 Aug 9; 5(17):8467-76.

Sheng Q, Wang M, Zheng J. A novel hydrogen peroxide biosensor based on enzymatically induced deposition of polyaniline on the functionalized graphenecarbon nanotube hybrid materials. Sensors Actuators B Chem [Internet]. Available from: http://www.sciencedirect.com/science/article /pii/S0925400511008306

Shulga YM, Baskakov SA, Abalyaeva VV, Efimov ON, Shulga NY, Michtchenko A, et al. Composite material for supercapacitors formed by polymerization of aniline in the presence of graphene oxide nanosheets. J Power Sources. 2013 Feb 15; 224(0):195-201.

Wan L, Wang S, Wang X, Dong B, Xu Z, Zhang X, et al. Room-temperature fabrication of graphene films on variable substrates and its use as counter electrodes for dye-sensitized solar cells. Solid State Sci. 2011 Feb; 13(2):468-75.

Wang G, Zhuo S, Xing W. Graphene/polyaniline nanocomposite as counter electrode of dye-sensitized solar cells. Mater Lett. 2012 Feb 15; 69:27-9.

Wang L, Ye YJ, Lu XP, Wen ZB, Li Z, Hou HQ, et al. Hierarchical Nanocomposites of Polyaniline Nanowire Arrays on Reduced Graphene Oxide Sheets for Supercapacitors. Sci Rep [Internet]. 2013. Available from: http:/ /www.nature.com/srep/2013/131220/ srep03568/full/srep03568.html.

Wehling TO, Novoselov KS, Morozov SV, Vdovin EE, Katsnelson MI, Geim AK, et al. Molecular Doping of Graphene. Nano Lett. 2008 Jan 1; 8(1):173-7. 